\title{
Measurement uncertainty due to work-piece error interaction with sampling period
}

\author{
Robert Edgeworth and Robert G. Wilhelm \\ Center for Precision Metrology \\ Department of Mechanical Engineering and Engineering Science \\ The University of North Carolina at Charlotte \\ 9201 University City Blvd., Charlotte, NC 28223-0001
}

Key words: metrology, uncertainty, coordinate measurement, sampling

Abstract: Dimensional measurements based upon point sampling contain theoretically infinite uncertainties in the intervals between sample locations. In practice, the uncertainty is bounded due to material and process constraints which produce an effective minimum spatial frequency for surface errors. In this paper we develop a relation for measurement uncertainty due to workpiece form error using the ratio between the spatial frequency of workpiece errors and the sampling period. Several applications of this calculation are briefly discussed.

\section{INTRODUCTION}

Commonly, sculptured surfaces are measured with the assumption that $[1,2]$ manufacturing errors are uniform over the surface. However, tool wear, machine inaccuracies, vibration, cutting forces, and velocity errors all contribute non-uniformly to surface errors. Just as these errors influence design function, non-uniform manufacturing errors also affect measurement uncertainty or the dispersion that reasonably can be attributed to the measurand.

Sculptured surfaces typically are measured with coordinate metrology methods that collect representative points from a surface of interest on the workpiece. These points are used to estimate substitute geometry which is often used to determine conformance of the sample piece to the design intent. As with any measurement, it is desirable for the user of this information to know the uncertainty associated with measurement quantities. Methods for determining the uncertainty associated with individual point measurements are well known and commonly used in practice today [3]. However, when a set of points are combined to assess a surface problems arise. When only a small group of points are used to evaluate a continuous surface, workpiece errors between sampling locations can result in large discrepancies between the calculated, substitute surfaces and the true surface. This paper develops an upper bound for the uncertainty that results from the interaction of workpiece errors and the spatial frequency of the sampling pattern. This result can be applied to make an estimate of the total uncertainty related to a specific measurement process.

It has long been recognized that insufficient samples are being taken in practice to account for the effect of workpiece form errors [4]. Form errors are particularly important when evaluating tolerances based upon bounded zones such as form and profile [5-9]. The uncertainty due to workpiece errors between sample points is theoretically infinite, although practically bounded by material and process constraints. For the purpose of metrology, we are most interested in the magnitude of the errors present on a workpiece. The Nyquist ratio gives a guideline for the frequencies of errors which can be detected by a given sampling interval, but does not provide guidance on how to sample in order to detect the full amplitude of the error signals.

Weckenmann et Al. [10], note that a good sampling procedure should permit the user to know the measurement uncertainty associated with the process. In most cases, such an

The original version of this chapter was revised: The copyright line was incorrect. This has been corrected. The Erratum to this chapter is available at DOI: 10.1007/978-0-387-35392-0_40 
uncertainty estimate is impossible to develop without making assumptions on the shape and distribution of workpiece form errors.

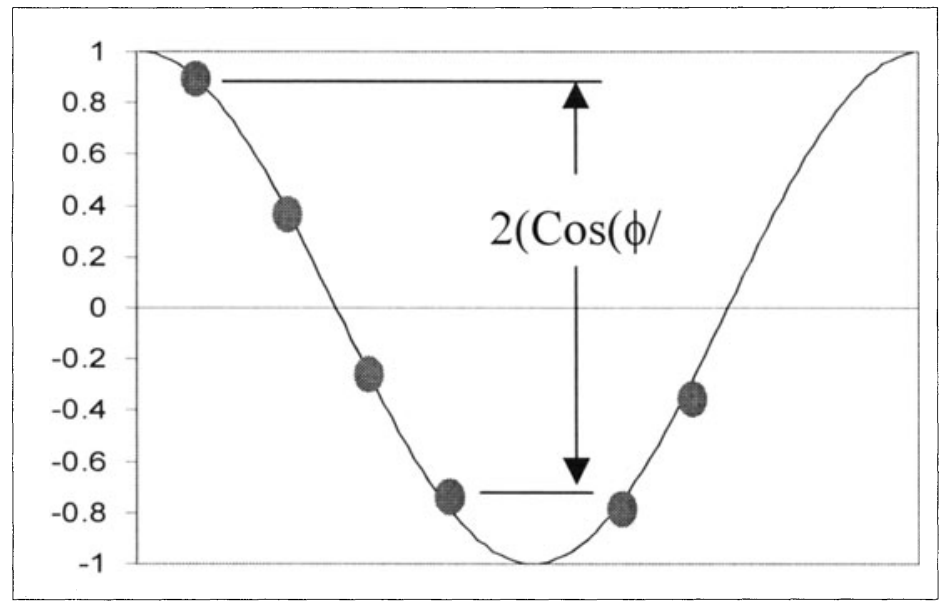

Figure 1. Maximum Measuring Error with Even Sampling Ratio

Recently, we have been working on using measurement of surface normals as well as position to help predict the behavior of surfaces between sample points. The surface normal data allows us to develop a cubic interpolating curve between pairs of sample points. This interpolant is capable of detecting $\mathrm{N}$ waves with $\mathrm{N}+1$ samples, a considerable improvement over position only data. We use the interpolated error curve across a section iteratively to select sample points until a set of measurement requirements are satisfied. This algorithm permits a measurement application to detect automatically all form errors up to a known frequency with a known uncertainty attributable to the sampling method $[11,12]$. It is difficult to compare the attributes of various sampling plans in metrology applications without determining the effect that workpiece shape errors can contribute to the measurement uncertainty.

Table 1. List of Symbols

\begin{tabular}{cl}
\hline L & Error Wavelength \\
\hline$\phi$ & Sample Spacing \\
$\mathrm{S}$ & Workpiece Size \\
$\mathrm{N}$ & Sample Size \\
$\mathrm{M}$ & Set of Points \\
$\omega$ & Frequency \\
$\theta$ & Phase Angle \\
$\mathrm{L} / \phi$ & Sampling Ratio \\
\hline
\end{tabular}

\section{PROBLEM FORMULATION}

Shape errors of sculptured surfaces are generally divided into 3 different types, based upon the wavelength of the error. In order of decreasing wavelength, the types are named form, waviness, and surface roughness. Form errors represent the longest error wavelengths with, typically, as many as 100 full waves on a surface. Waviness errors are repeated structures on a part surface, with up to 1000 complete waves on a part. Surface roughness 
errors are the very smallest structures on a surface; typically surface roughness would be considered as features with wavelengths smaller than $1 \mathrm{~mm}$. Generally, coordinate measurement techniques cannot detect surface roughness errors due to the mechanical filtering properties of the measuring instrument.

We wish to find the uncertainty due to workpiece shape errors as a function of the ratio between the sampling interval and the wavelength of the workpiece errors. It is assumed that the workpiece errors are non-random. We will first examine the simple case of a constant frequency, sinusoidal error shape and then extend our results to more complex shapes.

Consider a workpiece with a sinusoidal error imposed upon it of wavelength $L$ that is sampled uniformly at points spaced $\phi$ apart. The region of interest on the workpiece is s units long, and it is assumed the wavelength of errors present is less than the workpiece size. The number of samples collected is.

$$
\mathbf{N}=\mathbf{I N T}(\mathbf{s} / \phi)
$$

Then, we have the set of measurements $(\mathrm{M})$.

$$
\mathbf{M}=\{\theta, \theta+\phi, \ldots, \theta+(\mathbf{N}-\mathbf{1}) \phi\}
$$

Where $\theta$ is the initial phase relationship between the sampling pattern and the error signal. If the error amplitude is $\mathrm{A}$, the fractional sampling error is:

The set $M$ that maximizes the fractional sampling error contains the largest measurement error due to workpiece error that is possible for a given sampling ratio. This value is the

$$
\text { Error }=\frac{A-(\operatorname{Maximum}\{M\}-\operatorname{Minimum}\{M\})}{A}
$$

maximum uncertainty due to workpiece shape error.

A closed form solution for the workpiece uncertainty is not possible, but we can find an exact solution for the special case of even sampling ratios. Consider an error signal represented by a cosine wave with $2 \mathrm{n}$ sample points uniformly distributed across it. Symmetry causes the sample points to be equidistant from the maximum and minimum points of the error curve. The maximum measuring error occurs when the maximum value measured is $\cos (\phi / 2 \mathrm{~L})$ and the minimum measured value is $-\cos (\phi / 2 \mathrm{~L})$ (Figure 1$)$.

Therefore, the fractional uncertainty due to workpiece errors can be shown to be:

\section{$1-\operatorname{Cos}(\phi / 2 \mathrm{~L})$}

for even sampling ratios.

Interpolating between even values of the sampling ratio, we can develop a chart showing the relationship between the sampling ratio and the uncertainty due to workpiece shape errors (Figure 2). The uncertainty is equal to $100 \%$ of the shape error at the Nyquist rate of 2 samples per form error wave and decreases rapidly with increasing sample ratio.

This result can be shown to be a bounding function for combinations of trigonometric functions as well. Consider the combination of 2 sine waves of arbitrary phase:

$$
\mathbf{A}_{1} \sin \omega_{1}\left(\theta+\alpha_{1}\right)+\mathbf{A}_{2} \sin \omega_{2}\left(\theta+\alpha_{2}\right)
$$

There are 2 basic cases that need to be considered in this situation.

$$
\begin{aligned}
\omega_{1} & >\omega_{2} \quad \text { or; } \\
\omega_{1} & \approx \omega_{2}
\end{aligned}
$$

In the case of widely separated frequencies, the 2 signals are largely independent and easily can be treated independently. When 2 signals are closely matched in frequency they produce a beat signal at a frequency equal to the difference between the 2 input frequencies. The spatial size of the beat peaks and valleys are proportional to the signal input frequency. Therefore, our uncertainty model will be applicable to the base input frequencies of the signals. 


\section{DISCUSSION}

Figure 2 dramatically illustrates the influence workpiece shape errors can have upon the accuracy of dimensional measurements. To limit workpiece uncertainty to less than $5 \%$ of the magnitude of the shape errors, it is necessary to sample at 10 times the spatial frequency of those errors. Since typical feature evaluations in coordinate metrology involve the collection of very small numbers of sample points, it is clear that current practice is detecting accurately only the longest error wavelengths present on the workpiece.

This estimate of workpiece uncertainty is conservative since it does not account for process knowledge that could be used to sample consistently near the areas where the largest errors can be expected to occur. For example, a flat surface whose dominant error mode is the result of gravity bending generally will show the largest errors in the region around the center of the part. A well planned measurement will sample in this region and greatly reduce the influence of workpiece shape error upon the measuring uncertainty.

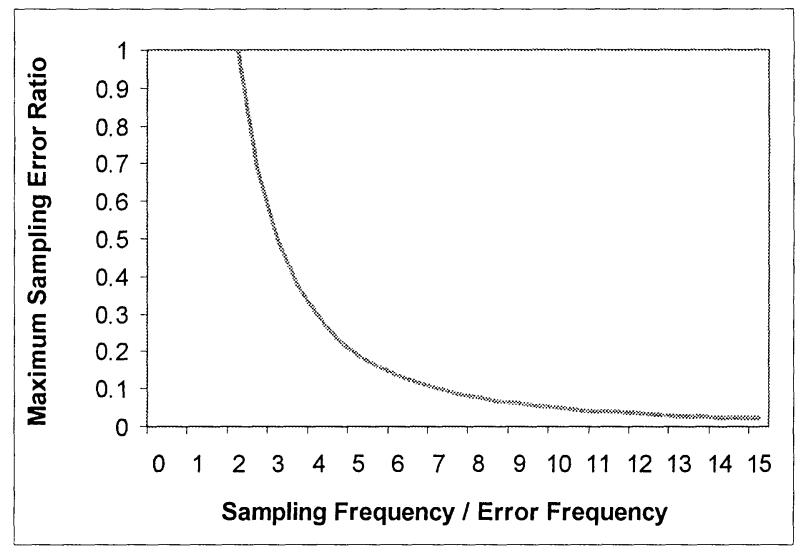

Figure 2. Fractional Error due to workpiece form error as a function of sampling ratio

The result presented here could be applied to improve measurement reliability in two ways. Firstly, the uncertainty chart could be used with process knowledge of the typical error frequencies that need to be controlled to determine an acceptable sample spacing during the measurement process planning stage. Another potential application would be to post process individual measurement results by means of a Fourier transform to determine the detectable error frequencies present upon the part. This information then could be combined with the sampling interval to calculate the uncertainty of the specific measurement. The result would be an uncertainty estimate supplied to the operator of the measurement system that is based upon the actual shape errors detected on each specific part. Measurements that resulted in an unacceptable high uncertainty could be repeated with a denser sampling pattern to ensure a reliable assessment of the part condition.

\section{CONCLUSIONS}

We have shown an upper limit for the uncertainty due to workpiece shape errors based upon the ratio between the error wavelengths and the sampling interval. This result can be applied to help select the sampling plan during the measurement process planning stage or as a post measurement calculation of uncertainty. 


\section{ACKNOWLEDGEMENTS}

This work has benefited greatly from the exceptional research environment provided by the Center for Precision Metrology at UNC Charlotte and the Cameron Center for Applied Research. This material is based upon work supported by the National Science Foundation under Grant No. DMI-9457168, the UNC Charlotte Center for Precision Metrology Research Affiliates and the National Science and Engineering Research Council of Canada.

\section{REFERENCES}

1. C.-H. Menq, H.-T. Yau, and G.-Y. Lai, Automated Precision Measurement of Surface Profile in CADDirected Inspection, IEEE Journal of Robotics and Automation, 8(2):268-278, 1992

2. Tuckey, C., Finding Small Rotations and Translations in Imperfect Part and Die Models, Journal of Manufacturing Systems, 11(6):442--448, 1992

3. Phillips, S. D., "Chapter 7: Performance Evaluations", Coordinate Measuring Machines and Systems, J. A. Bosch, Ed., Marcel Dekker Inc., New York, 1995.

4. Hocken, R. J., Raja, J., Babu, U., "Sampling Issues in Coordinate Metrology", Manufacturing Review, 1993, 6 (4), 282 - 294.

5. Kim, N. and Kim S., "Geometrical Tolerances: Improved Linear Approximation of Least Squares Evaluation of Circularity by Minimum Variance", Int. J Mach. Tools Manufact., 1996, 36 (3), 355366.

6. Cheragi, S., Huay S., and Motavalli S., "Straightness and Flatness Tolerance Evaluation: an Optimization Approach", Precision Engineering, 1996, 18, 30-37.

7. Carr, K. and Ferriera, P., "Verification of Form Tolerance Part I: Basic Issues, Flatness and Straightness", Precision Engineering, 1995, 17,131-143.

8. Carr, K. and Ferriera, P., "Verification of Form Tolerance Part II: Cylindricity and Straightness of a Median Line", Precision Engineering, 1995, 17, 144-156.

9. Lai, J. and Chen I., "Minimum Zone Evaluation of Circles and Cylinders", Int. J. Mach. Tools Manufact. 1996, 35 (4), 435-451.

10. Weckenmann, A., Eitzert, H., Garmer, M., and Weber, H., "Functionality-oriented evaluation and sampling strategy in coordinate metrology", Precision Engineering, 1995, 17, 244-252.

11. Edgeworth R. and Wilhelm R.G, "An Iterative Approach to Profile Evaluation Using Interpolation of Measured Positions and Surface Normals", In H.A. ElMaraghy, editor, Geometric Design Tolerancing: Theories, Standards and Applications, H.A. ElMaraghy, Ed., Chapman \& Hall, 1998, ISBN 0-412-83000-0.

12. Edgeworth R. and Wilhelm R.G, "Adaptive Sampling for Coordinate Metrology", Accepted for publication, Precision Engineering 